\title{
Tuning Viral Capsid Nanoparticle Stability with Symmetrical Morphogenesis
}

Aida Llauró ${ }^{\ddagger}$ Benjamin Schwarz $z^{\ddagger}$ Ranjit Koliyatt, Pedro J. de Pablo, Trevor Douglas

\begin{tabular}{c|cc|cc}
\hline & \multicolumn{2}{|c|}{ S5 } & \multicolumn{2}{c}{ S3/S2 } \\
& $\begin{array}{c}\text { \# of } \\
\text { particles }\end{array}$ & $\%$ & $\begin{array}{c}\text { \# of } \\
\text { particles }\end{array}$ & $\%$ \\
\hline EX & 29 & 59 & 20 & 41 \\
\hline EX+Dec & 37 & 70 & 16 & 30 \\
\hline WB & 25 & 66 & 13 & 34 \\
\hline WB+Dec & 34 & 68 & 16 & 32 \\
\hline
\end{tabular}

Table S1. Adsorption frequency depending on the adsorption symmetry. The number of single particles and its corresponding percentage are shown. 


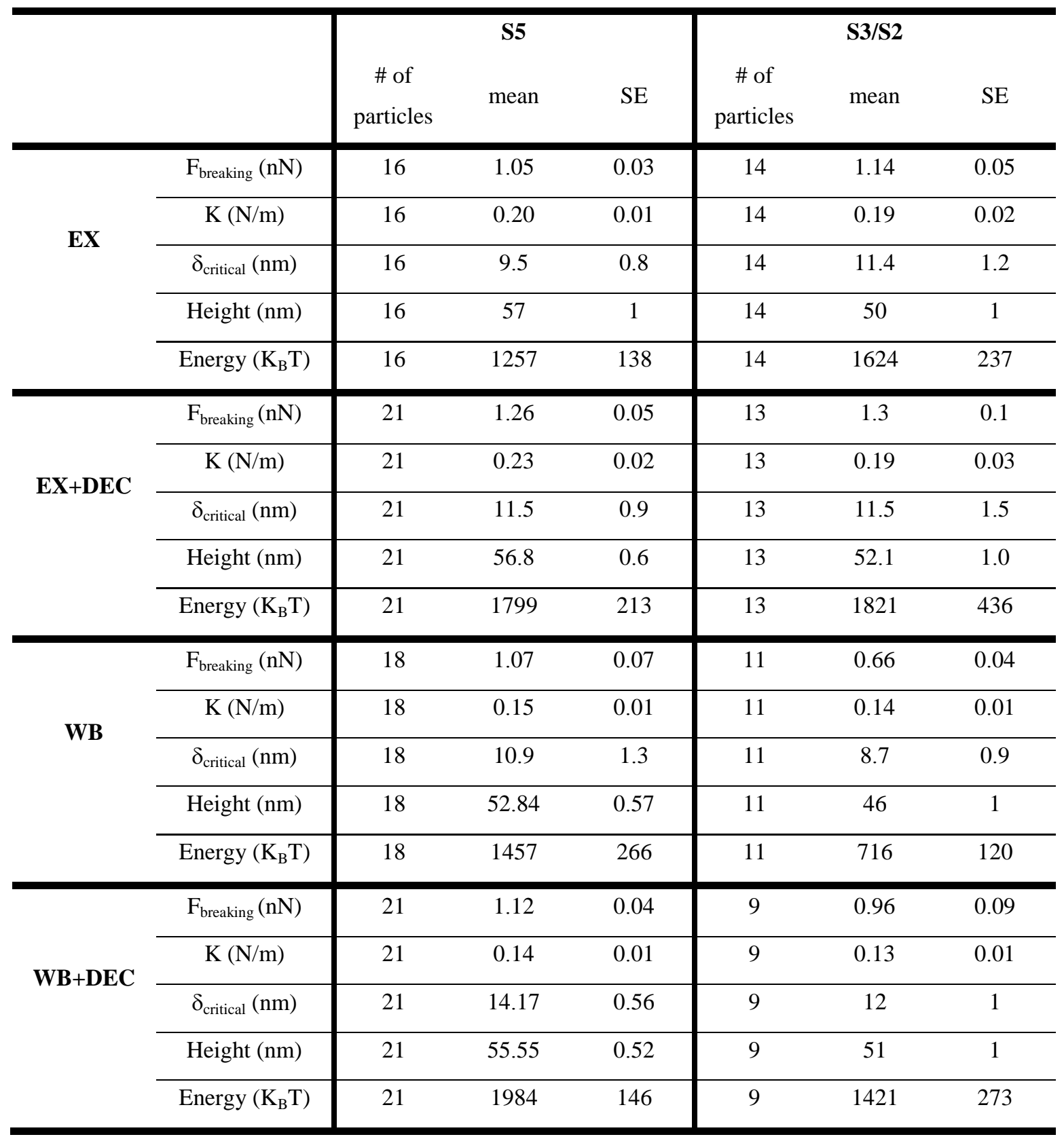

Table S2. Mechanical characterization. Breaking force, elastic constant $(\mathrm{k})$, critical indenation $\left(\delta_{\text {critical }}\right)$ and height for the different morphologies: EX, EX+Dec, WB and WB+Dec. SE: standard error. 

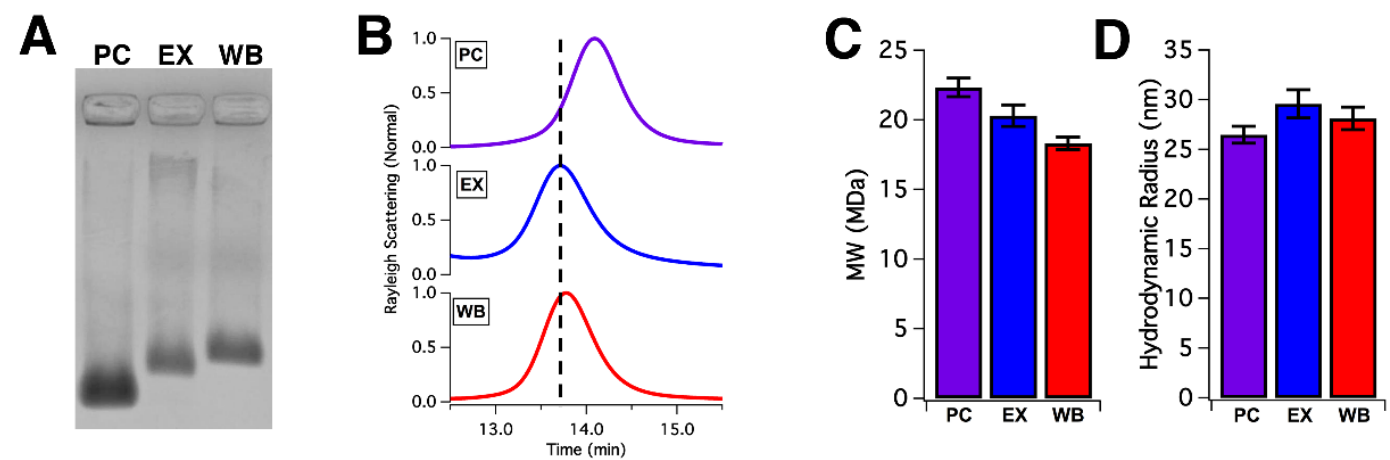

Figure S1. Characterization of capsids shows complete expansion of EX and subsequent penton loss in WB. (A) Non-denaturing agarose gel shift of PC, EX and WB capsid samples. (B) SEC chromatograms of $\mathrm{PC}, \mathrm{EX}$ and WB displaying the Rayleigh scattering ratio. Dashed line marks the retention time of EX and WB.

(C) Number average molecular weight of PC, EX and WB samples measured by multi-angle light scattering. Error bars represent the standard error on the mean. (D) Average hydrodynamic radius of PC, EX and WB measured across the peak width at half max of the SEC chromatograms.
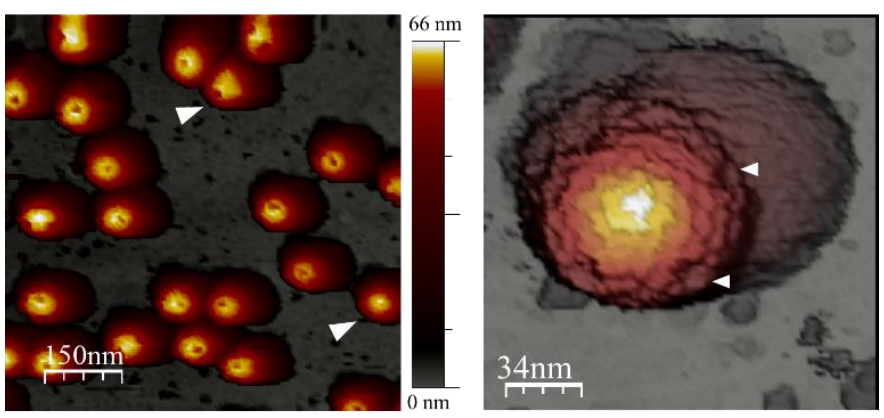

Figure S2. AFM images of WB presenting some of the pentons. (left) Large AFM view. The white arrowheads indicate a particles adsorbed along the 3- and 5-fold symmetry axes showing some pentons. (right) High-resolution of a WB with a penton on the top. The arrowheads indicate two penton vacancies. 
A

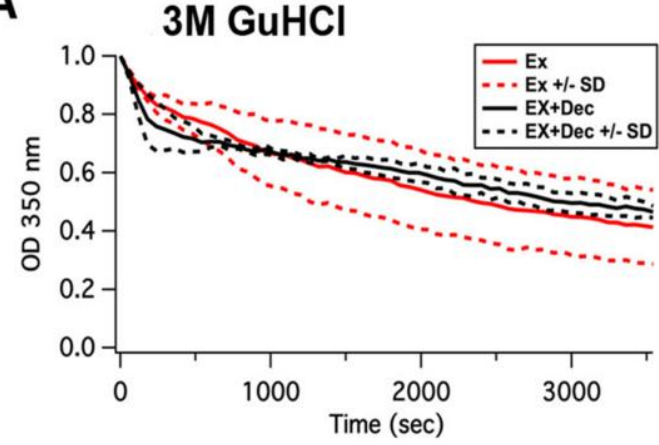

C

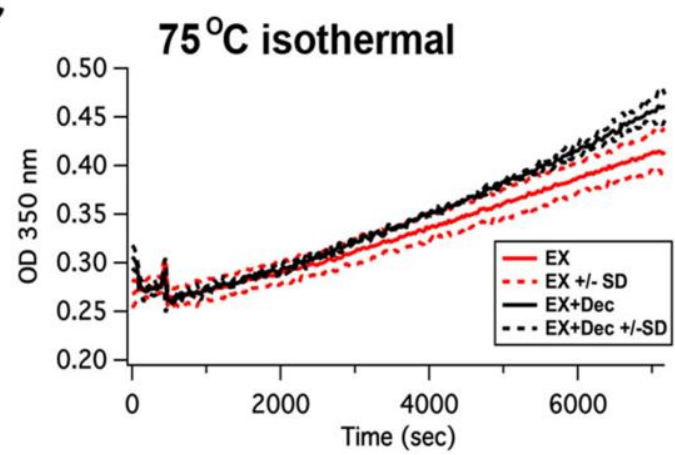

B

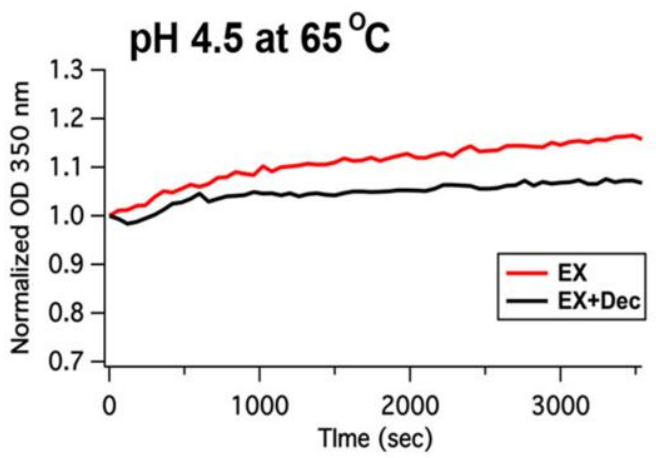

D

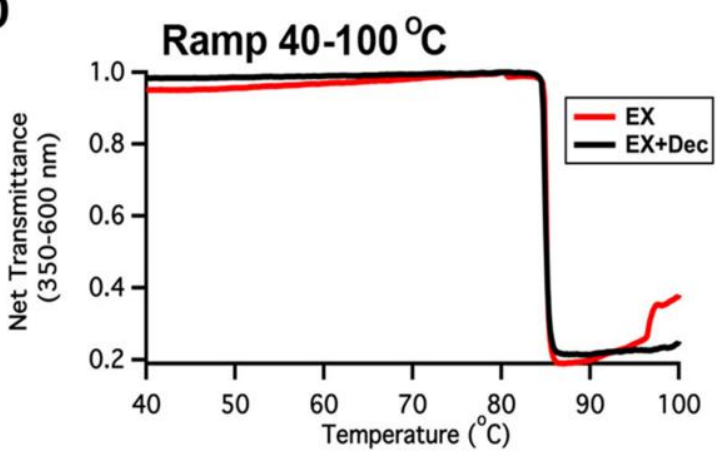

Figure S3. Chemical and thermal stressors have either no effect on the capsid morphologies or fail to resolve differences in stability between morphologies. (A) P22 EX with (black) and without (red) Dec disassembled in the presence of $3 \mathrm{M} \mathrm{GuHCl}$. Dashed lines represent + or - one standard deviation. (B) P22 EX with and without Dec in the presence of $\mathrm{pH} 4.5$ incubated at $65^{\circ} \mathrm{C}$. (C) P22 EX with and without Dec incubated at 75 ${ }^{\circ} \mathrm{C}$. Increase in scattering was deemed to be due to evaporation and aggregation was not evident in solution. Samples were run $n=3$, dashed lines represent + or - one standard deviation. (D) EX with and without Dec in increasing temperature at a rate of $1{ }^{\circ} \mathrm{C} / \mathrm{min}$ using a Luma $40^{\mathrm{TM}}$ peltier heating unit (Quantum Northwest) 

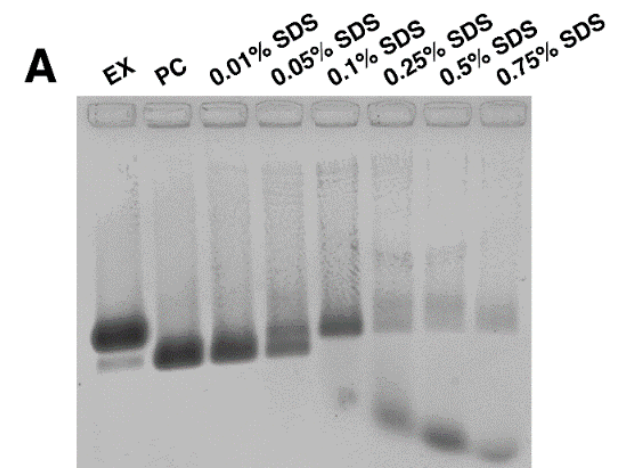

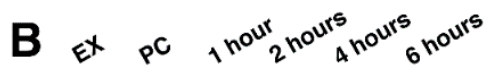

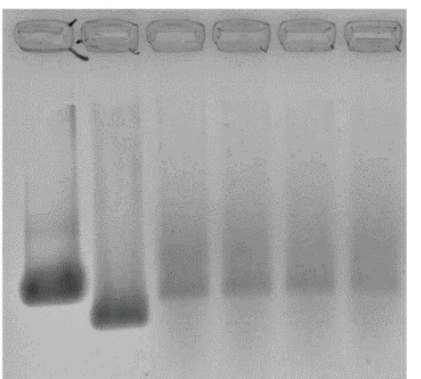

Figure S4. SDS expands and then degrades the P22 VLP. (A) PC VLP incubated in increasing concentrations of SDS for 15 minutes. (B) Extended incubation of PC in $0.1 \%$ SDS. In both gels a PC and heat-expanded EX control are included for reference.

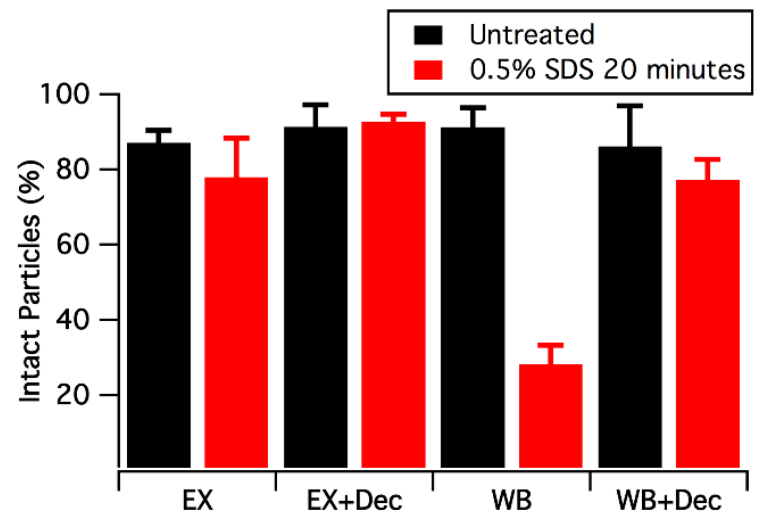

Figure S5. Percentage of undamaged structures observed by TEM. 


\begin{tabular}{cccccc}
\hline \multicolumn{2}{c}{ EX } & \multicolumn{3}{c}{ WB } \\
\hline Interface & Symmetry & $\begin{array}{c}\text { Buried Surface Area } \\
\left(\AA^{2}\right)\end{array}$ & Interface & Symmetry & $\begin{array}{c}\text { Buried Surface Area } \\
\left(\AA^{2}\right)\end{array}$ \\
\hline F1-G1 & Q-3 & 833.3 & C7-E1 & Q-3 & 1384.2 \\
B1-C10 & Q-3 & 809.2 & B1-C10 & Q-3 & 1222.3 \\
C7-E1 & Q-3 & 797.2 & D1-D7 & I-3 & 1200.5 \\
A1-G2 & Q-3 & 770.9 & B1-E2 & Q-3 & 1182.2 \\
B1-E2 & Q-3 & 751.8 & B1-C1 & Q-6 & 928.0 \\
D7-E1 & Q-2 & 609.0 & E1-F1 & Q-6 & 922.7 \\
A1-F2 & Q-3 & 533.7 & A1-B1 & Q-6 & 832.7 \\
B1-F2 & Q-2 & 525.9 & A1-F2 & Q-3 & 794.1 \\
A1-G1 & Q-2 & 511.7 & D1-E1 & Q-6 & 757.3 \\
C1-C10 & I-2 & 502.0 & C1-D1 & Q-6 & 721.5 \\
D1-D7 & I-3 & 462.8 & A1-F1 & Q-6 & 471.7 \\
B1-C1 & Q-6 & 421.2 & & & \\
A1-F1 & Q-6 & 311.7 & & & \\
C1-D1 & Q-6 & 292.8 & & & \\
E1-F1 & Q-6 & 267.5 & & & \\
\hline
\end{tabular}

Table S3. Association energy between inter- and intra capsomeric subunits for EX and WB. The association energy was extracted from ViperdB. ${ }^{1}$ In black are represented interaction existing in both forms, in red and blue interfaces lost due to the transition, and in green interactions gained due to the transition. Note that red corresponds to interactions with the pentons (subunit $\mathrm{G}$ ) and blue to interactions between hexamers. All the gained interactions were within an hexamer subunit (green) (see Figure S6 for position of the interfaces). 

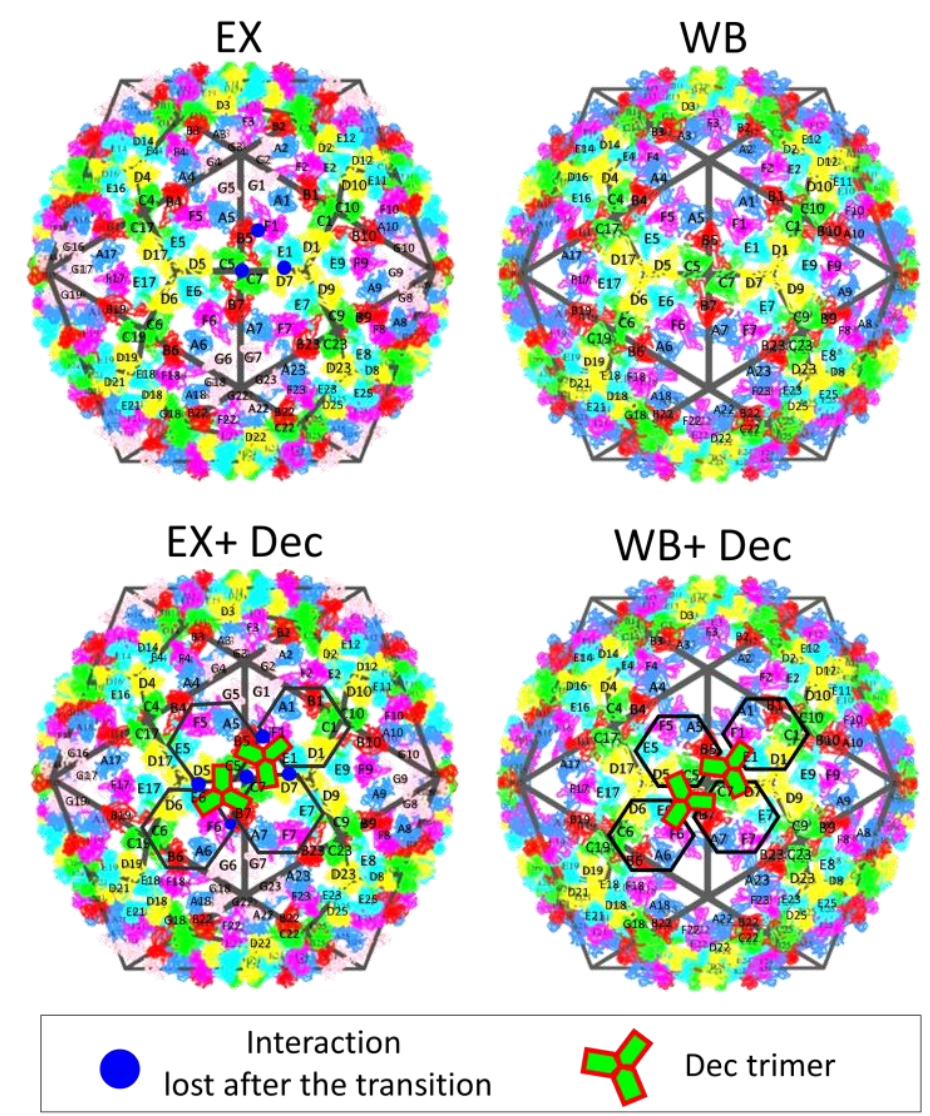

Figure S6. Interaction energy between subunits (based on table S3). Hexameric subunits (A-G) are colored in blue, red, green, yellow, cyan and pink, respectively. Pentameric subunits $(\mathrm{G})$ are colored in pale pink. (top) Comparison of EX and WB forms (PDB: 3iyi and 3iyh) ${ }^{2}$. Blue dots in EX represent the interfaces where the interactions were lost after the transition. (bottom) Comparsion of EX+Dec and WB+Dec. The position of two Dec trimer are superimposed on the structures; each trimer lays between three hexamers (marked with a solid black line $)^{3,4}$. Note that the trimer reinforces the position where the interactions were lost after the transition. 

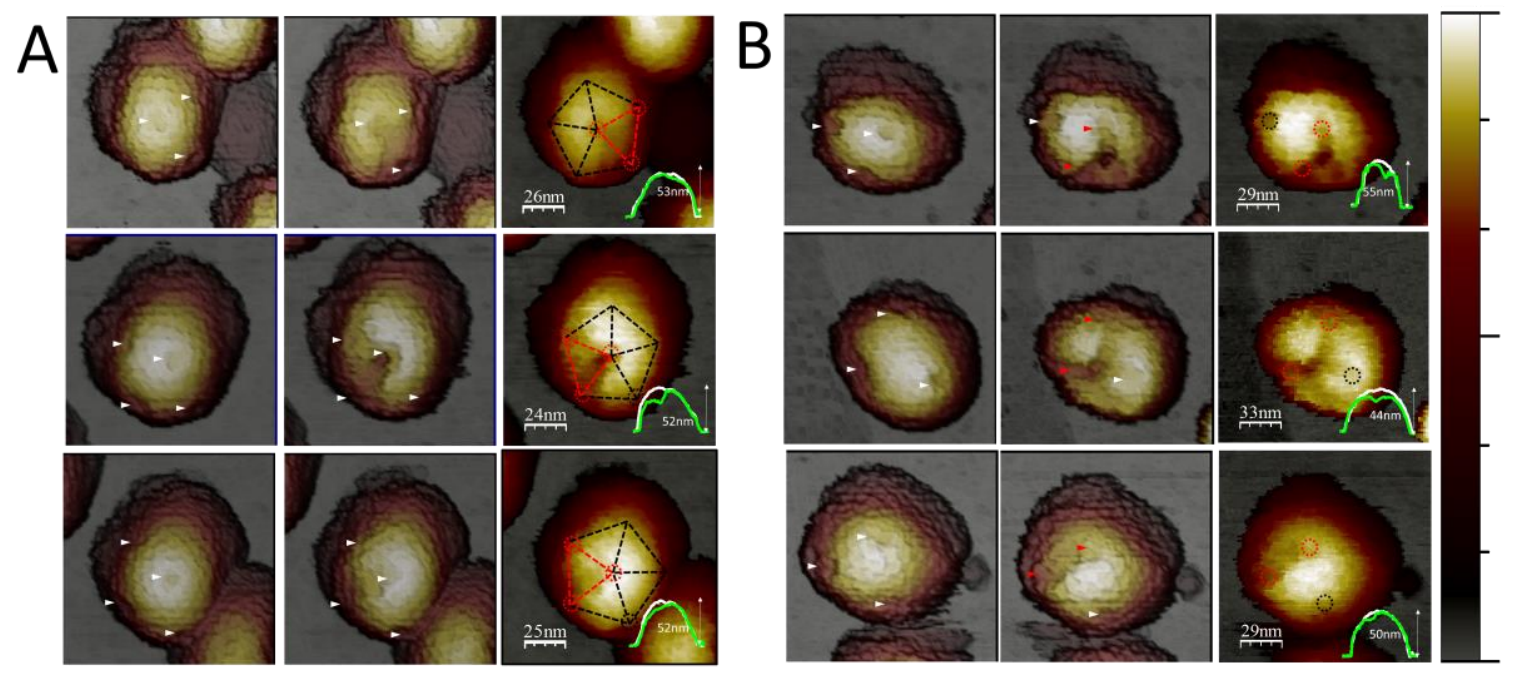

Figure S7. Pattern of breakage. (A) Each row: 3D AFM image of an WB capsid in the S5 axes before and after the nanoindentation (left and middle). The image on the right side shows a 2D representation of the particle after the breakage. A pentagon with its 5 faces is overlapped to the structure (dash lines). The red lines highlighted the parts of the particles that were clearly affected. (inset) Profile of the particle before (white) and after (green line) the damage. (B) As before but with three WB capsids along their S3 axes. In the 2D AFM images (right) the circles indicate the penton vacancies. Color scale bar was normalized for each individual particle to the maximum height (see inset).

\section{REFERNCES}

1. $\quad$ Carrillo-Tripp, M.; Shepherd, C. M.; Borelli, I. A.; Venkataraman, S.; Lander, G.; Natarajan, P.; Johnson, J. E.; Brooks, C. L., 3rd; Reddy, V. S. VIPERdb2: an Enhanced and Web API Enabled Relational Database for Structural Virology. Nucleic Acids Res 2009, 37, D436-42.

2. $\quad$ Parent, K. N.; Khayat, R.; Tu, L. H.; Suhanovsky, M. M.; Cortines, J. R.; Teschke, C. M.; Johnson, J. E.; Baker, T. S. P22 Coat Protein Structures Reveal a Novel Mechanism for Capsid Maturation: Stability without Auxiliary Proteins or Chemical Crosslinks. Structure 2010, 18, 390-401.

3. Parent, K. N.; Deedas, C. T.; Egelman, E. H.; Casjens, S. R.; Baker, T. S.; Teschke, C. M. Stepwise Molecular Display Utilizing Icosahedral and Helical Complexes of Phage Coat and Decoration Proteins in the Development of Robust Nanoscale Display Vehicles. Biomaterials 2012, 33, 5628-5637.

4. $\quad$ Tang, L.; Gilcrease, E. B.; Casjens, S. R.; Johnson, J. E. Highly Discriminatory Binding of Capsid-Cementing Proteins in Bacteriophage L. Structure 2006, 14, 837-845. 